\title{
Características otorrinolaringológicas en niños con trisomía 21: un estudio de 171 pacientes en el Hospital Infantil de México Federico Gómez
}

\author{
Natalia Peraza, Carlos De La Torre, Alicia Álvarez y Perla Villamor* \\ Servicio de Otorrinolaringología Pediátrica, Hospital Infantil de México Federico Gómez, Ciudad de México, México
}

\begin{abstract}
Resumen
Introducción: Los niños con trisomía 21 enfrentan una amplia gama de problemas en la región de la cabeza y el cuello, por lo cual es importante reconocer las manifestaciones otorrinolaringológicas que presentan, así como su apropiado manejo. Métodos: Estudio de serie de casos retrospectivo de pacientes pediátricos con trisomía 21. De cada caso se analizó el espectro de manifestaciones otorrinolaringológicas, el manejo establecido y los resultados. Resultados: Se incluyeron 171 niños. La edad media de la primera valoración por otorrinolaringología en la institución fue de $7.2 \pm 4.2$ años. Las manifestaciones otológicas más frecuentes fueron la estenosis del conducto auditivo externo y la disfunción de la trompa de Eustaquio. Más de la mitad de los pacientes (63\%) presentaron hipoacusia, principalmente de tipo conductivo bilateral, y hasta el $75 \%$ de los pacientes con afectación otológica requirieron algún procedimiento quirúrgico. Las manifestaciones rinológicas más comunes fueron la rinosinusitis crónica y la rinitis alérgica. La apnea obstructiva del sueño estuvo presente en el $30 \%$ de los pacientes. El tratamiento principal fue la amigdalectomía, seguida del tratamiento con dispositivos de presión positiva de la vía aérea. Menos del $5 \%$ de los pacientes presentaron un compromiso laríngeo. Conclusiones: Los pacientes pediátricos con trisomía 21 deben ser remitidos sistemáticamente a una evaluación otorrinolaringológica periódica, debido a la alta incidencia de manifestaciones en esta región. Se deben ofrecer tratamientos oportunos para mejorar su salud y calidad de vida.
\end{abstract}

Palabras clave: Trisomía 21. Síndrome de down. Niños. Otorrinolaringología.

\section{Otolaryngological features in children with trisomy 21: a study of 171 patients at the Hospital Infantil de México Federico Gómez}

\begin{abstract}
Introduction: Children with trisomy 21 face a wide range of conditions in the head and neck region, for which it is important that physicians are aware and have a strong understanding of the ear, nose, and throat (ENT) disorders, and their management as well. Methods: Retrospective case series of pediatric patients with trisomy 21. The spectrum of otolaryngological manifestations, their management, and outcomes of each case were analysed. Results: One hundred and seventeen pediatric patients were included. The mean age was $7.2 \pm 4.2$ years. More than half of the patients (63\%) had hearing loss (HL). The most frequent presentation was conductive $H L$, predominating the mild and bilateral type. The most common otological
\end{abstract}

Correspondencia:

*Perla Villamor

E-mail: perla.villamor@gmail.com
Disponible en internet: 18-03-2019 Bol Med Hosp Infant Mex. 2019;76:87-94

www.bmhim.com

1665-1146/৫ 2019. Hospital Infantil de México Federico Gómez, impreso por Permanyer México SA de CV, todos los derechos reservados. 
manifestations found were external ear canal stenosis and Eustachian tube dysfunction. Up to $75 \%$ of the patients with otologic involvement required some surgical procedure. The most common rhinological manifestations were chronic rhinosinusitis and allergic rhinitis. Obstructive sleep apnea (OSA) was present in $30 \%$ of all patients, which main treatment was tonsillectomy, followed by continuous positive and biphasic positive airway pressure treatments. Less than $5 \%$ of the patients presented a laryngeal compromise. Conclusions: Pediatric patients with trisomy 21 systematically should be referred to periodic ENT assessment due to the high incidence of manifestations in this region. Timely treatments should be offered in order to improve the health and the quality of life of the patient.

Key words: Trisomy 21. Down syndrome. Children. Otolaryngology

\section{Introducción}

La trisomía 21 es uno de los trastornos genéticos más comunes y la causa más importante de discapacidad intelectual ${ }^{1}$. Fue descrito por primera vez en 1866 por John L. Down. Es más habitualmente causado por un error de no disyunción, que resulta en tres copias del cromosoma $21^{2}$. La incidencia de la trisomía 21 se ve afectada por la edad de la madre y varía en la población (entre 1 en 319 y 1 en 1,000 nacidos vivos) ${ }^{3}$. Tiene una alta variabilidad fenotípica y complejidad genética ${ }^{4}$. La mejora reciente en el manejo médico ha mejorado la expectativa de vida de los pacientes con síndrome de Down, por lo que en países desarrollados se presenta un promedio de vida de 55 años ${ }^{5}$.

Varias características de la trisomía 21 se encuentran en la región de la cabeza y el cuello y pueden causar secuelas que impiden que los pacientes alcancen sus máximas capacidades de desarrollo, además de obstaculizar su calidad de vida ${ }^{6}$. A medida que avance la ciencia médica y estos pacientes vivan más tiempo, es importante que los médicos tengan conciencia y una sólida comprensión de las manifestaciones otorrinolaringológicas de los niños con síndrome de Down, así como de su manejo.

El objetivo de este estudio fue presentar una serie de casos de pacientes pediátricos con trisomía 21, su espectro de manifestaciones otorrinolaringológicas, características clínicas y sociodemográficas, manejo establecido y resultados.

\section{Métodos}

Se llevó a cabo un estudio retrospectivo de serie de casos.

\section{Recopilación de datos}

Se creó una base de datos con registros médicos de pacientes pediátricos ( $<18$ años $)$ con trisomía 21 confirmada por cariotipo, valorados en el Servicio de Otorrinolaringología del Hospital Infantil de México Federico Gómez durante el periodo de enero de 2007 a enero de 2017.

Se registraron los siguientes datos para cada paciente: características demográficas, enfermedades sistémicas asociadas, diagnóstico otorrinolaringológico primario y secundario, manejo quirúrgico recibido, resultados posquirúrgicos y complicaciones. El audiograma más completo y temprano se utilizó para caracterizar la audición y, en casos de pérdida auditiva, para determinar el tipo, la gravedad y la lateralidad de la misma. Dentro del protocolo de la institución, los pacientes menores de 2 años son evaluados mediante potenciales evocados auditivos de tallo (PEATC) por vía ósea y vía aérea. A mayor edad son evaluados mediante audiometría tonal por vía aérea y vía ósea por juego u observación del comportamiento. Pacientes mayores a 2 años en quienes no es posible la realización de la audiometría continúan con evaluación por PEATC.

\section{Gestión de datos y estadísticas}

Los datos se recopilaron en formularios y se analizaron utilizando Stata Statistical Software, versión 13 (College Station, TX, StataCorp LP). Las variables continuas se presentan como la media \pm desviación estándar y las variables categóricas se presentan como recuentos.

\section{Ética}

Este estudio se llevó a cabo de acuerdo con la Declaración de Helsinki de la Asociación Médica Mundial sobre protocolos y ética en investigación médica.

\section{Resultados}

Se incluyeron 171 pacientes pediátricos con trisomía 21 confirmada por evaluación genética y cariotipo. Entre la población de estudio, la edad media en el momento 
Tabla 1. Características de los pacientes

\begin{tabular}{|l|c|}
\hline Característica & $\mathbf{N}=\mathbf{1 7 1} \mathbf{n}(\%)$ \\
\hline $\begin{array}{l}\text { Edad en el momento de la primera } \\
\text { valoración por otorrinolaringología } \\
\text { HIMFG (años } \pm \text { SD) }\end{array}$ & $7.1 \pm 4.2$ \\
\hline $\begin{array}{l}\text { Sexo } \\
\text { Masculino }\end{array}$ & \\
\hline Femenino & $98(57.3)$ \\
\hline Morbilidad asociada & $73(42.7)$ \\
\hline Sobrepeso/Obesidad & \\
Cardiopatía & $139(81.3)$ \\
Hipotiroidismo & $124(72.5)$ \\
Diabetes & $48(28.1)$ \\
Labio hendido o paladar hendido & $4(2.4)$ \\
Leucemia linfoblástica aguda & $4(2.4)$ \\
\hline
\end{tabular}

HIMFG: Hospital Infantil de México Federico Gómez; SD: desviación estándar.

de la primera valoración por el servicio de otorrinolaringología fue de $7.2 \pm 4.2$ años (rango: 1 mes a 18 años). El sexo femenino representó el $42.7 \%$ de los casos, con una relación de 1:1.4 (Tabla 1). La morbilidad asociada principalmente incluía sobrepeso/obesidad, enfermedad cardiovascular e hipotiroidismo, seguidos de diabetes, labio y paladar hendido y leucemia linfoblástica aguda (Tabla 1).

En cuanto a los hallazgos audiológicos, el $63 \%$ de los pacientes presentaban pérdida de audición. La presentación más frecuente fue hipoacusia conductiva (92\% de los casos), predominando el tipo leve y bilateral. Por otro lado, la hipoacusia neurosensorial representó solamente el $18 \%$ de los casos, aunque con una presentación más grave. La hipoacusia bilateral profunda fue el tipo más común de hipoacusia neurosensorial. Del total de pacientes, el $15.05 \%$ (14 pacientes) fueron rehabilitados con adaptación de auxiliares auditivos. Sin embargo, no fue posible establecer los casos en los que la adaptación se realizó de forma uni o bilateral. Ninguno de los pacientes se rehabilitó mediante sistemas Cross (por sus siglas en inglés, contralateral routing of signal) o Bi-Cross, dispositivos de conducción ósea ni implante coclear (Tabla 2).

La principal patología del oído externo fue la estrechez del conducto auditivo externo (CAE), la cual se presentó en el $98.24 \%$ de los pacientes, con un espectro que varió desde una estrechez parcial hasta la estenosis. Adicionalmente, la estrechez del CAE fue el único compromiso otológico en el $46 \%$ de los pacientes. Las manifestaciones otológicas más comunes encontradas después de la estenosis del CAE fueron la disfunción de la trompa de Eustaquio sin derrame y la
Tabla 2. Manifestaciones, tratamientos y procedimientos para el manejo de patología otológica

\begin{tabular}{|c|c|}
\hline Característica & $N=171 \mathrm{n}(\%)$ \\
\hline $\begin{array}{l}\text { Patología de oído externo } \\
\text { Conducto auditivo externo estrecho } \\
\text { Microtia }\end{array}$ & $\begin{array}{c}168(98.25) \\
18(10.52)\end{array}$ \\
\hline $\begin{array}{l}\text { Patología de oído medio } \\
\text { Disfunción de la trompa de Eustaquio sin } \\
\text { derrame } \\
\text { Otitis media con derrame } \\
\text { Otitis media crónica sin colesteatoma } \\
\text { Otitis media crónica con colesteatoma }\end{array}$ & $\begin{array}{l}39(22.81) \\
37(21.64) \\
11(6.43) \\
6(3.51)\end{array}$ \\
\hline $\begin{array}{l}\text { Hallazgos audiológicos } \\
\text { Audición normal } \\
\text { Total hipoacusia de cualquier tipo } \\
\text { Hipoacusia conductiva } \\
\text { Leve } \\
\text { Moderada } \\
\text { Grave } \\
\text { Profunda } \\
\text { Bilateral } \\
\text { Unilateral }\end{array}$ & $\begin{array}{c}62(36.26) \\
109(63.74) \\
92(53.8) \\
60(35.09) \\
32(18.71) \\
0(0) \\
0(0) \\
83(48.54) \\
9(5.26)\end{array}$ \\
\hline $\begin{array}{l}\text { Hipoacusia neurosensorial } \\
\text { Leve } \\
\text { Moderada } \\
\text { Grave } \\
\text { Profunda } \\
\text { Bilateral } \\
\text { Unilateral }\end{array}$ & $\begin{array}{c}18(10.52) \\
2(1.17) \\
0(0) \\
3(1.75) \\
12(7.02) \\
12(7.02) \\
5(2.92)\end{array}$ \\
\hline $\begin{array}{l}\text { Tratamiento* } \\
\text { Manejo médico } \\
\text { Adaptación de auxiliares auditivos } \\
\text { Miringotomía con colocación de tubo de } \\
\text { ventilación } \\
\text { Miringotomía sin colocación de tubo de } \\
\text { ventilación } \\
\text { Mastoidectomía de muro alto } \\
\text { Timpanoplastia } \\
\text { Adenoidectomía (para resolver problemas } \\
\text { otológicos) }\end{array}$ & $\begin{array}{c}23(24.7) \\
14(15.05) \\
37(39.8)^{\dagger} \\
15(16.1) \\
7(7.5) \\
6(6.5) \\
5(5.4)\end{array}$ \\
\hline Edad al momento de cirugía (años \pm SD) & $4.6 \pm 3.7$ \\
\hline
\end{tabular}

SD: desviación estándar.

* Porcentaje basado en el total de pacientes con patología otológica ( $N=93$ ). †Un total de 47 miringotomías con colocación de tubo de ventilación se realizaron en 37 pacientes. El promedio de tubos de ventilación por paciente fue de $1.3 \pm 0.48$, intervalo de confianza al $95 \%$ : $1.145-1.455$.

otitis media con derrame, seguida de otitis media crónica sin colesteatoma y la otitis media crónica con colesteatoma (Tabla 2). Hasta el $75 \%$ de los pacientes con afectación otológica requirieron algún procedimiento quirúrgico, mientras que el $25 \%$ restante se manejaron únicamente con tratamiento médico (ejercicios de trompa de Eustaquio, aerosol nasal, antibiótico sistémico y gotas óticas con quinolonas). La principal cirugía realizada para tratar la afectación otológica fue la miringotomía, con o sin colocación de tubo de 
Tabla 3. Manifestaciones nasosinusales

\begin{tabular}{|l|c|}
\hline Patología & N = 171 n (\%) \\
\hline Rinosinusitis crónica & $36(21.1)$ \\
\hline Rinitis alérgica & $33(19.3)$ \\
\hline Rinosinusitis fúngica invasiva & $2(1.2)$ \\
\hline $\begin{array}{l}\text { Intervención* } \\
\text { Manejo médico }\end{array}$ & $48(67.6)$ \\
\hline $\begin{array}{l}\text { Adenoidectomía (para manejo de la } \\
\text { patología nasosinusal) }\end{array}$ & $21(29.6)$ \\
\hline $\begin{array}{l}\text { Turbinoplastia } \\
\text { Cirugía endoscópica funcional }\end{array}$ & $8(11.3)$ \\
\hline Edad en el momento de cirugía (años \pm SD) & $2(2.8)$ \\
\hline
\end{tabular}

SD: desviación estándar.

*Porcentaje basado en el total de pacientes con patología nasosinusal $(\mathrm{N}=71)$.

ventilación, la cual se realizó en el $56 \%$ de los pacientes con compromiso otológico. Se realizaron, en promedio, $1.27 \pm 0.48$ miringotomías por paciente. La edad promedio de colocación de tubos de ventilación fue $3.81 \pm 2.87$ años (rango de 1 a 14 años). De los 52 pacientes que se sometieron a miringotomía, seis presentaron otorrea durante el seguimiento y dos tuvieron una perforación timpánica residual. La timpanoplastia, mastoidectomía y adenoidectomía (específica para el manejo de problemas otológicos) se realizaron en menos del $10 \%$ de los pacientes (Tabla 2).

Las manifestaciones nasosinusales más comunes fueron la rinosinusitis crónica y la rinitis alérgica. También se documentaron dos casos de rinosinusitis fúngica invasiva asociada a leucemia linfoblástica aguda. El principal tratamiento para el compromiso nasosinusal fueron lavados nasales, aerosol nasal, antihistamínicos y antibióticos sistémicos, así como la adenoidectomía (específica para el tratamiento de la rinosinusitis crónica). La cirugía endoscópica funcional (CEF) y la turbinoplastia se realizaron en menos del $10 \%$ de los casos de compromiso nasosinusal (Tabla 3).

La macroglosia fue la principal manifestación en la cavidad oral y la orofaringe, con una prevalencia del $69 \%$. Los trastornos respiratorios del sueño estuvieron presentes en el $30 \%$ de los pacientes. La hipertrofia amigdalina obstructiva fue evidente en el $24 \%$ de los casos. El tratamiento principal para los trastornos obstructivos del sueño fue la amigdalectomía, seguida del tratamiento médico, que consistió principalmente en el uso de dispositivos de presión positiva continua de la vía aérea (Cpap) y de presión positiva bifásica (Bi-Pap). El 11\% de los pacientes que se sometieron a amigdalectomía persistieron con síntomas obstructivos nocturnos a pesar del tratamiento quirúrgico, los cuales se definieron como pausas respiratorias presenciadas por el cuidador o un profesional de la salud, acompañadas de roncopatía. Por otro lado, la hemorragia tardía postamigdalectomía ocurrió en un paciente, que fue manejado satisfactoriamente con hemostasia en quirófano (Tabla 4).

Menos del $5 \%$ de los pacientes presentaron un compromiso laríngeo. Cuatro pacientes tenían laringomalacia, de los cuales tres fueron tratados satisfactoriamente con tratamiento médico y seguimiento. Solamente un paciente requirió supraglotoplastia. Dos pacientes presentaron estenosis subglótica adquirida que ameritó el aseguramiento de la vía aérea con traqueostomía y dilataciones laríngeas endoscópicas. Durante el seguimiento, uno de los dos pacientes fue decanulado con éxito (Tabla 5).

\section{Discusión}

Teniendo en cuenta que la plasticidad neural, el desarrollo del lenguaje y el inicio de la escolaridad ocurren antes de los 7 años de edad, la primera valoración por el servicio de otorrinolaringología se encontró a una edad poco favorable en esta serie de casos. Por ello, deben establecerse protocolos de derivación oportuna a un especialista en otorrinolaringología de forma rutinaria en los pacientes con diagnóstico o sospecha de trisomía 21. Las recomendaciones de diagnóstico y rehabilitación en hipoacusia realizadas por la Joint Committee on Infant Hearing indican un diagnóstico antes de los 3 meses de vida, con un inicio en la rehabilitación antes de los 6 meses, lo cual es aún más relevante en esta población que tiene una alta prevalencia de hipoacusia.

Los niños con trisomía 21 experimentan una prevalencia mayor de diagnósticos concomitantes en comparación con individuos sin el síndrome. Kong, et al. reportaron que las infecciones de las vías respiratorias superiores, la otitis media, los síntomas nutricionales, metabólicos y los retrasos globales del desarrollo se encontraban entre las comorbilidades más comunes en casos de síndrome de Down? ${ }^{7}$ En esta serie, el sobrepeso/obesidad, la enfermedad cardiovascular y el hipotiroidismo fueron las comorbilidades más prevalentes.

En cuanto a las manifestaciones otorrinolaringológicas, Lau, et al., en su estudio de 50 pacientes con síndrome de Down, encontraron que un tercio de los casos tenía algún tipo de hipoacusia, y de estos, el $72 \%$ eran de tipo conductivo ${ }^{8}$. Kreicher, et al., utilizando la base de datos audiológica y genética AudGenDB de 
Tabla 4. Manifestaciones en la cavidad oral y faringe

\begin{tabular}{|l|c|}
\hline Patología & $\mathbf{N}=171$ n (\%) \\
\hline $\begin{array}{l}\text { Macroglosia } \\
\text { Hipertrofia amigdalina }\end{array}$ & $118(69)$ \\
Síntomas de trastorno respiratorio del sueño sin PSG diagnóstica & $41(24)$ \\
\hline AOS confirmada por PSG & $40(23.4)$ \\
\hline $\begin{array}{l}\text { Intervención* } \\
\text { Manejo médico incluyendo dispositivos de presión positiva de vía aérea (Cpap/Bi-Pap) } \\
\text { Amigdalectomía }\end{array}$ & $11(6.4)$ \\
\hline $\begin{array}{l}\text { Complicaciones } \\
\quad \text { Persistencia de síntomas obstructivos postamigdalectomía }\end{array}$ & $15(29.4)$ \\
\hline Sangrado postamigdalectomía & $36(70.6)$ \\
\hline Edad al momento de cirugía (años \pm SD) & $4(11.1)$ \\
\hline
\end{tabular}

AOS: apnea obstructiva del sueño; PSG: polisomnografía; SD: desviación estándar.

${ }^{*}$ Porcentaje basado en el total de pacientes con AOS $(N=51)$.

tPorcentaje basado en el total de amigdalectomías $(N=36)$.

Tabla 5. Manifestaciones laríngeas

\begin{tabular}{|l|c|}
\hline Patología & N = 171 n (\%) \\
\hline Laringomalacia & $4(2.3)$ \\
\hline Estenosis subglótica & $2(1.2)$ \\
\hline Intervención* & \\
$\quad$ Manejo médico & $3(50)^{\dagger}$ \\
\hline Traqueostomía & $2(33.3)$ \\
Dilatación endoscópica laríngea & $2(33.3)$ \\
\hline Supraglotoplastia & $1(16.7)$ \\
\hline Edad al momento de cirugía (años \pm SD) & $4.8 \pm 6.2$ \\
\hline
\end{tabular}

SD: desviación estándar.

* Porcentaje basado en el total de pacientes con patología laríngea $(N=6)$.

tSolo pacientes con laringomalacia.

1,088 pacientes con trisomía 21, encontraron que el $67 \%$ de los pacientes cursaba con déficit de audición que persistía en todos los audiogramas disponibles ${ }^{9}$. En el presente estudio, el $63.7 \%$ de los pacientes tenía algún tipo de pérdida auditiva, que era principalmente conductiva y de tipo bilateral y leve. Sin embargo, en casos de pérdida auditiva neurosensorial, el compromiso fue predominantemente profundo y bilateral. La Academia Estadounidense de Pediatría recomienda que un niño con trisomía 21 que aprobó su prueba de detección neonatal debe someterse a estudios electrofisiológicos de audición cada 6 meses hasta que se pueda realizar una audiometría de tono puro (generalmente después de los 4 años) y, posteriormente, una audiometría anual de seguimiento ${ }^{10}$. Es posible que la baja incidencia de hipoacusia neurosensorial en la presente serie, así como la aparente ausencia de hipoacusias mixtas, se deban al método de detección empleado en pacientes mayores de 2 años (audiometría por juego $u$ observación del comportamiento), el cual podría subestimar, en algunos casos, un componente neurosensorial leve o una hipoacusia unilateral con oído contralateral normoacúsico. Se debe tener en cuenta que la evaluación auditiva es un desafío en esta población, puesto que los niños con síndrome de Down pueden tener poca atención durante las pruebas de comportamiento y una respuesta voluntaria inconsistente, lo que dificulta diferenciar el tipo y el lado de la hipoacusia. Por lo tanto, las pruebas electrofisiológicas, como los potenciales evocados auditivos de tallo cerebral, son particularmente útiles en este contexto, ya que pueden evaluar el rendimiento auditivo, independientemente de la edad y el estado mental del paciente ${ }^{8}$.

En esta serie, el $15.05 \%$ de los pacientes fueron rehabilitados con adaptación de auxiliares auditivos, aunque no fue posible establecer los casos en los que la adaptación se realizó de forma uni o bilateral. Ninguno de los pacientes se rehabilitó mediante sistemas Cross o Bi-Cross, dispositivos de conducción ósea o implante coclear. El uso de audífonos convencionales es complejo en estos pacientes, ya que muchos de estos niños tienen problemas de aprendizaje y no cooperan con un dispositivo en el oído. Además, las ayudas convencionales pueden exacerbar la otitis externa y la otitis media coexistente ${ }^{11}$. Un estudio reciente mostró que los niños con síndrome de Down tuvieron una mejora significativa en sus actividades diarias, aprendizaje y concentración con dispositivos de conducción ósea como el BAHA®. El dispositivo BAHA® también mejoró el desarrollo de habilidades auditivas y redujo el lapso 
de distracción en estos pacientes, por lo cual se considera en la rehabilitación auditiva después de que los audífonos convencionales han fallado ${ }^{11}$.

La mayoría de los niños con trisomía 21 tienen una afección otológica, que incluye canales auditivos estrechos, otitis media crónica y pérdida de audición que exige evaluación y tratamiento por un otorrinolaringó$\log 0^{12}$. En el presente estudio, el $46 \%$ de los pacientes tenían estenosis del CAE y el $54 \%$ presentaban otro tipo de compromiso otológico adicional. La estenosis del CAE se ha descrito previamente en el 40 a $50 \%$ de los pacientes con síndrome de Down, lo que produce una impactación de cera, pelos y cuerpos extraños difíciles de eliminar que contribuyen a la hipoacusia conductiva $^{13,14}$. Aunque no se ha establecido una definición universalmente aceptada de estenosis, un estudio de pacientes con estenosis aural congénita la definió como un diámetro del canal $\leq 4 \mathrm{~mm}^{15}$. En un estudio reciente de 130 pacientes con trisomía 21, Dy y Lapeña determinaron que la tasa de prevalencia de estenosis del conducto auditivo en la población estudiada era del $72.3 \%{ }^{16}$.

La dismorfia facial conduce a anomalías del oído y de las vías respiratorias superiores, incluidos los canales auditivos estenóticos y la disfunción de la trompa de Eustaquio ${ }^{17}$. Este fenotipo, combinado con una función inmunitaria deficiente, resulta en el desarrollo de infecciones de las vías respiratorias superiores ${ }^{18}$. Además, la hipotonía generalizada puede conducir a una disfunción del músculo tensor del velo palatino del paladar, lo que aumenta el riesgo de otitis media aguda y derrame crónico por colapso de la trompa de Eustaquio ${ }^{19}$. Cuando la disfunción de trompa de Eustaquio permanece sin diagnosticar, pueden aparecer secuelas tardías, como perforación timpánica, bolsas de retracción y colesteatoma. Se ha descrito que el $7.1 \%$ de los pacientes con trisomía 21 desarrollan colesteatoma durante el seguimiento ${ }^{20,21}$. En esta serie, solo el 3\% de los pacientes tenían otitis media crónica con colesteatoma y todos se trataron con éxito con una mastoidectomía de muro alto. Cabe señalar que no se reportó ninguna mastoidectomía de muro bajo en los pacientes estudiados. El retraso en el diagnóstico y tratamiento de un colesteatoma podría provocar una fístula laberíntica, laberintitis, parálisis del nervio facial y complicaciones endocraneales ${ }^{20,21}$.

Crowson, et al., en su serie de casos de 676 niños con disfunción de la trompa de Eustaquio, incluidos 46 con trisomía 21, reportaron que la fluticasona intranasal aumentó el tiempo para la colocación de un tubo de ventilación; sin embargo, este hallazgo no aplicó para los niños con síndrome de Down ${ }^{22}$. En la presente serie, la cirugía principal realizada para tratar la afectación otológica fue la miringotomía con o sin colocación de tubo de ventilación (56\% de los casos). Esta cirugía es el procedimiento quirúrgico pediátrico más común en todo el mundo. Sin embargo, la inserción del tubo de ventilación puede ser difícil o imposible en niños con trisomía 21, ya que las características morfológicas incluyen canales del oído muy angostos. Es posible que una intervención más temprana, con tubos de ventilación en niños con otitis media con efusión, pueda conducir a mejores resultados al reducir las cicatrices y el daño permanente de la audición; sin embargo, se necesita un estudio prospectivo para evaluar esta posibilidad. Además, se ha demostrado que la colocación quirúrgica de tubos de ventilación mejora significativamente la calidad de vida. En este estudio, el número promedio de miringotomías por paciente fue de $1.27 \pm 0.48$. Uno de los riesgos de la colocación repetida de los tubos de ventilación es la incidencia del $17 \%$ de perforaciones de la membrana timpánica ${ }^{23}$. Estos pacientes también corren el riesgo de perforación permanente después de la extrusión del tubo, lo cual se debe a una membrana timpánica muy delgada que carece de lámina propia, una capa rica en vasos sanguíneos y fibras de colágeno ${ }^{13}$. En la presente serie, seis de los 52 pacientes que se sometieron a miringotomía presentaron otorrea durante el seguimiento y dos tuvieron una perforación timpánica residual manejada con éxito con timpanoplastia. Ghadersohi, et al. demostraron recientemente una tasa de éxito de la timpanoplastia global del $70.9 \%$ (tasa de cierre inicial del $54.8 \%$ ). El tipo de tubo, específicamente uno de acción prolongada (tubo-T), no se asoció con la perforación persistente basada en su análisis. Los autores abogaron por la timpanoplastia de cartílago en esta población para resistir mejor la presión negativa del oído medio generada por la trompa de Eustaquio ${ }^{23}$. Los dispositivos de amplificación podrían usarse para mejorar la audición, pero los audífonos retroauriculares convencionales con frecuencia no son tolerados ${ }^{18}$. Por otro lado, se ha demostrado que la adenoidectomía aumenta el beneficio de la inserción de tubos de ventilación en ciertos grupos de niños sin trisomía $21^{24}$.

Los pacientes con trisomía 21 pueden sufrir sinusitis recurrente debido a un desarrollo anormal en los senos frontales, maxilares y esfenoides. La hipoplasia y la no neumatización total son comunes en esta población. Por otro lado, la maduración retrasada del sistema inmunitario o la discinesia ciliar también pueden causar sinusitis crónica ${ }^{14}$. En este estudio, se detectaron la 
rinosinusitis crónica y la rinitis alérgica como manifestaciones rinológicas más comunes, con dos casos de rinosinusitis fúngica invasiva asociada con leucemia linfoblástica aguda. En pacientes con síndrome de Down, la rinorrea puede mejorar con la edad debido al agrandamiento de las anomalías anatómicas. El tratamiento debe ser similar al de otros casos, incluidos los antibióticos, los esteroides nasales, los descongestivos y los antihistamínicos. Si la sinusitis persiste, las alternativas quirúrgicas pueden incluir la adenoidectomía y la cirugía endoscópica sinusal funcional ${ }^{13}$. En este estudio, la gran mayoría de los pacientes se trataron con éxito solo con tratamiento médico y solo en el $2.8 \%$ de los casos se realizó la CEF.

La macroglosia fue la principal manifestación en la cavidad oral y la orofaringe, con una prevalencia del $69 \%$. Los trastornos respiratorios del sueño estuvieron presentes en el $30 \%$ de los pacientes. La apnea obstructiva del sueño (AOS) es una comorbilidad frecuente en esta población, con una prevalencia estimada entre el 30 y el $70 \%{ }^{25}$. En un metaanálisis reciente, Lee, et al. encontraron que las prevalencias de AOS basadas en un índice de apnea hipoapnea $(\mathrm{IAH})>1,1.5$, 2, 5 y 10 eventos/h fueron del $69 \%, 76 \%, 75 \%, 50 \%$ y $34 \%$, respectivamente, en los niños con trisomía 21 que se sometieron a polisomnografía. Los análisis de subgrupos no revelaron diferencias significativas ${ }^{26}$. Las características craneofaciales, incluida la hipoplasia maxilar, la macroglosia y la hipotonía oral orofaríngea, en general, contribuyen a la alteración del sueño durante la respiración. Los niños con síndrome de Down tienen un alto riesgo de AOS, no solo por las diferencias morfológicas, sino también por su tendencia a la obesidad. Por otro lado, las anomalías endocrinológicas múltiples, como el hipotiroidismo y la diabetes mellitus tipo 1, están asociadas con el aumento de peso y la obesidad ${ }^{27}$. En la presente serie, solo el $6.4 \%$ de los pacientes tenían una polisomnografía diagnóstica (PSG). La baja frecuencia de realización de PSG podría deberse al alto costo del estudio, lo que genera un subdiagnóstico de AOS en esta serie. La PSG en el laboratorio se considera el estándar de oro para el diagnóstico de la AOS pediátrica, aunque el costo y la disponibilidad a menudo limitan su uso. Ante la dificultad de realizar PSG en todos los pacientes por altos costos y accesibilidad, sería recomendable considerar estudios alternativos, aunque no sean estándar de oro. Un ejemplo es la poligrafía respiratoria o la endoscopia bajo sueño inducido, que implica un costo mucho menor para valorar oportunamente la obstrucción de estos pacientes antes de realizar alguna cirugía, determinando adicionalmente el nivel de obstrucción de la vía aérea superior. Lo anterior es relevante si se tiene en cuenta que la obstrucción puede ser multinivel o diferente a la sospechada solamente con el examen físico. La realización de este estudio es posible durante la inducción anestésica con laringoscopia flexible previa a un procedimiento quirúrgico programado ${ }^{28}$.

Farhood, et al. mostraron una mejoría estadísticamente significativa en los valores de PSG entre los pacientes con trisomía 21 y AOS que se sometieron a una amigdalectomía en una revisión sistemática. Sin embargo, la mayoría de los pacientes no pudieron lograr un $\mathrm{IAH}<1^{29}$. En el presente estudio, la tasa de persistencia de síntomas obstructivos después de la amigdalectomía fue del $11 \%$. Sin embargo, al no contar con polisomnografía de rutina pre y posquirúrgica, es posible que se esté subestimando el porcentaje real de AOS residual postamigdalectomía. En general, mediante la realización de una polisomnografía se puede esperar una reducción del $51 \%$ en el IAH preoperatorio con la intervención de la adenoamigdalectomía. Esta es información útil para aconsejar y manejar la expectativa de los padres. También es un recordatorio para los médicos acerca de la importancia de seguir de cerca a esta población de pacientes después de la cirugía y, aunque se pueden observar mejorías, los pacientes con síndrome de Down a menudo presentarán enfermedad persistente y podrán requerir más cirugías o una intervención con dispositivos de presión positiva Cpap/Bi-Pap.

Las anomalías de las vías respiratorias se describen como más prevalentes en pacientes con trisomía 21. En un estudio, el $13.8 \%$ de los niños con trisomía 21 tenían anomalías en las vías respiratorias, cuyos diagnósticos más comunes fueron la traqueobroncomalacia y la estenosis subglótica ${ }^{30}$. Según lo reportado en la literatura, los niños con trisomía 21 tienen al menos el doble de probabilidades de presentar laringomalacia en comparación con niños no sindrómicos. Además, casi el $50 \%$ puede mostrar un colapso epiglótico en la endoscopia del sueño. Sin embargo, en la presente serie, menos del $5 \%$ de los pacientes presentaron un compromiso laríngeo. Se considera que la baja tasa de afectación laríngea reportada podría deberse a la falta de una búsqueda sistemática de manifestaciones laríngeas en pacientes aparentemente asintomáticos en esta área. Con respecto al tratamiento, cuatro pacientes de esta serie cursaron con laringomalacia, de los cuales tres fueron manejados satisfactoriamente con tratamiento médico y seguimiento; solo un paciente requirió supraglotoplastia, que fue satisfactoria. 
El presente estudio permite evidenciar la variedad de patologías otorrinolaringológicas de los pacientes pediátricos con trisomía 21, su morbilidad y la repercusión en su calidad de vida. Todos los pacientes pediátricos con síndrome de Down deberían ser remitidos sistemáticamente a evaluación por un especialista en otorrinolaringología, debido a la alta incidencia de las manifestaciones del síndrome en oídos, nariz y garganta. Se deben ofrecer tratamientos oportunos para mejorar su calidad de vida y permitir una mayor inclusión social a través de la mejora de las habilidades de comunicación, el control de la patología infecciosa y la reducción de la estancia hospitalaria.

\section{Responsabilidades éticas}

Protección de personas y animales. Los autores declaran que para esta investigación no se han realizado experimentos en seres humanos ni en animales.

Confidencialidad de los datos. Los autores declaran que han seguido los protocolos de su centro de trabajo sobre la publicación de datos de pacientes.

Derecho a la privacidad y consentimiento informado. Los autores han obtenido el consentimiento informado de los pacientes o sujetos referidos en el artículo. Este documento obra en poder del autor de correspondencia.

\section{Conflicto de intereses}

Los autores no declaran ningún conflicto de intereses.

\section{Bibliografía}

1. Asim A, Kumar A, Muthuswamy S, Jain S, Agarwal S. Down syndrome: an insight of the disease. J Biomed Sci. 2015;22:41.

2. Kanamori G, Witter M, Brown J, Williams-Smith L. Otolaryngologic manifestations of Down syndrome. Otolaryngol Clin North Am. 2000;33:1285-92.

3. Carothers AD, Hecht CA, Hook EB. International variation in reported live birth prevalence rates of Down syndrome, adjusted for maternal age. J Med Genet. 1999;36:386-93.

4. Mégarbané A, Ravel A, Mircher C, Sturtz F, Grattau Y, Rethoré MO, et al. The $50^{\text {th }}$ anniversary of the discovery of trisomy 21 : the past, present, and future of research and treatment of Down syndrome. Genet Med. 2009;11:611-6.

5. Glasson EJ, Sullivan SG, Hussain R, Petterson BA, Montgomery PD, Bittles $\mathrm{AH}$, et al. The changing survival profile of people with Down syndrome: implications for genetic counselling. Clin Genet. 2002;62:390-3.

6. Chin CJ, Khami MM, Husein M. A general review of the otolaryngologic manifestations of Down syndrome. Int J Pediatr Otorhinolaryngol. 2014; 78:899-904.
7. Kong AM, Hurley D, Evans KA, Brixner D, Csoboth C, Visootsak J. $A$ retrospective, longitudinal, claims-based comparison of concomitant diagnoses between individuals with and without Down syndrome. J Manag Care Spec Pharm. 2017;23:761-70.

8. Lau WL, Ko CH, Cheng WW. Prevalence and parental awareness of hearing loss in children with Down syndrome. Chin Med J (Engl). 2015; 128:1091-5.

9. Kreicher KL, Weir FW, Nguyen SA, Meyer TA. Characteristics and progression of hearing loss in children with Down syndrome. J Pediatr. 2018; 193:27-33.

10. Bull MJ; Committee on Genetics. Health supervision for children with Down syndrome. Pediatrics. 2011;128:393-406.

11. Mc Dermott AL, Williams J, Kuo MJ, Reid AP, Proops DW. The role of bone anchored hearing aids in children with Down syndrome. Int $\mathrm{J} \mathrm{Pe}$ diatr Otorhinolaryngol. 2008;72:751-7.

12. Shott SR, Joseph A, Heithaus D. Hearing loss in children with Down syndrome. Int J Pediatr Otorhinolaryngol. 2001;61:199-205.

13. Ramia M, Musharrafieh U, Khaddage W, Sabri A. Revisiting Down syndrome from the ENT perspective: review of literature and recommendations. Eur Arch Otorhinolaryngol. 2014;271:863-9.

14. Shott SR. Down syndrome: common otolaryngologic manifestations. Am J Med Genet C Semin Med Genet. 2006;142C:131-40.

15. Cole RR, Jahrsdoerfer RA. The risk of cholesteatoma in congenital aural stenosis. Laryngoscope. 1990;100:576-8.

16. Dy AES, Lapeña JFF Jr. External auditory canal dimensions, age, and cerumen retention or impaction in persons with Down syndrome. Ann Otol Rhinol Laryngol. 2018;127:253-7.

17. Bluestone C. Studies in otitis media: Children's Hospital of Pittsburgh, University of Pittsburgh Progress Report-2004. Laryngoscope. 2004; 114:1-26.

18. Fortnum $\mathrm{H}$, Leighton $\mathrm{P}$, Smith MD, Brown L, Jones M, Benton $\mathrm{C}$, et al. Assessment of the feasibility and clinical value of further research to evaluate the management options for children with Down syndrome and otitis media with effusion: a feasibility study. Health Technol Assess. 2014;18:1-147.

19. Rodman R, Pine HS. The otolaryngologist's approach to the patient with Down syndrome. Otolaryngol Clin North Am. 2012;45:599-629.

20. Bacciu A, Pasanisi E, Vincenti V, Giordano D, Caruso A, Lauda L, et al. Surgical treatment of middle ear cholesteatoma in children with Down syndrome. Otol Neurotol. 2005;26:1007-10.

21. lino $Y$, Imamura $Y$, Harigai S, Tanaka Y. Efficacy of tympanostomy tube insertion for otitis media with effusion in children with Down syndrome. Int J Pediatr Otorhinolaryngol. 1999;49:143-9.

22. Crowson MG, Ryan MA, Ramprasad VH, Choi KJ, Raynor E. Intranasal fluticasone associated with delayed tympanostomy tube placement in children with eustachian tube dysfunction. Int J Pediatr Otorhinolaryngol. 2017;94:121-6.

23. Ghadersohi S, Ida JB, Bhushan B, Billings KR. Outcomes of tympanoplasty in children with down syndrome. Int J Pediatr Otorhinolaryngol. 2017; 103:36-40

24. MRC Multicentre Otitis Media Study Group. Adjuvant adenoidectomy in persistent bilateral otitis media with effusion: hearing and revision surgery outcomes through 2 years in the TARGET randomised trial. Clin Otolaryngol. 2012;37:107-16.

25. Maris M, Verhulst S, Wojciechowski M, Van de Heyning P, Boudewyns A. Prevalence of obstructive sleep apnea in children with Down syndrome. Sleep. 2016;39:699-704.

26. Lee CF, Lee CH, Hsueh WY, Lin MT, Kang KT. Prevalence of obstructive sleep apnea in children with Down syndrome: A meta-analysis. J Clin Sleep Med. 2018;14:867-75.

27. Lal C, White DR, Joseph JE, van Bakergem K, LaRosa A. Sleep-disordered breathing in Down syndrome. Chest. 2015;147:570-9.

28. Heubi CH, Meinzen-Derr J, Shott SR, Smith DF, Ishman ASL. Polysomnography in pediatric otolaryngology: if not obstructive sleep apnea, what is it? Otolaryngol Head Neck Surg. 2017;157:1053-9.

29. Farhood Z, Isley JW, Ong AA, Nguyen SA, Camilon TJ, LaRosa AC, et al. Adenotonsillectomy outcomes in patients with Down syndrome and obstructive sleep apnea. Laryngoscope. 2017;127:1465-70.

30. Bertrand P, Navarro H, Caussade S, Holmgren N, Sánchez I. Airway anomalies in children with Down syndrome: endoscopic findings. Pediatr Pulmonol. 2003;36:137-41. 